\title{
Collagen XI sequence variations in nonsyndromic cleft palate, Robin sequence and micrognathia
}

\author{
Miia Melkoniemi ${ }^{1}$, Hannele Koillinen ${ }^{2,3}$, Minna Männikkö ${ }^{1}$, Matthew L Warman ${ }^{4}$, \\ Tero Pihlajamaa $^{1,5}$, Helena Kääriäinen ${ }^{6}$, Jorma Rautio ${ }^{7}$, Jyri Hukki ${ }^{7}$, Joseph A Stofko ${ }^{8}$, \\ George J Cisneros ${ }^{8}$, Deborah Krakow ${ }^{9,10,11}$, Daniel H Cohn ${ }^{9,12}$, Juha Kere ${ }^{2,13}$ \\ and Leena Ala-Kokko*,1,14
}

\begin{abstract}
${ }^{1}$ Collagen Research Unit, Biocenter and Department of Medical Biochemistry and Molecular Biology, University of Oulu, Oulu, Finland; ${ }^{2}$ Department of Medical Genetics, University of Helsinki, Helsinki, Finland; ${ }^{3}$ Department of Pediatric Neurology, University of Turku, Turku, Finland; ${ }^{4}$ Department of Genetics, Case Western Reserve School of Medicine, Cleveland, OH, USA; ${ }^{5}$ NMR Laboratory, Institute of Biotechnology, University of Helsinki, Helsinki, Finland;

${ }^{6}$ Department of Medical Genetics, The Family Federation of Finland, Helsinki, Finland; ${ }^{7}$ Cleft Center, Department of Surgery, Helsinki University Hospital, Helsinki, Finland; ${ }^{8}$ Montefiore Medical Center, Bronx, NY, USA; ${ }^{9}$ Ahmanson Department of Pediatrics, Steven Spielberg Pediatric Research Center, Los Angeles, CA, USA; ${ }^{10}$ Department of Obstetrics and Gynecology, Burns and Allen Cedars-Sinai Research Center, Los Angeles, CA, USA; ${ }^{11}$ Department of Obstetrics and Gynecology, Geffen School of Medicine at UCLA, Los Angeles, CA, USA; ${ }^{12}$ Departments of Human Genetics and Pediatrics, Geffen School of Medicine at UCLA, Los Angeles, CA, USA; ${ }^{13}$ Department of Biosciences at Novum and Clinical Research Centre, Karolinska Institute, Stockholm, Sweden; ${ }^{14}$ Center for Gene Therapy and Department of Medicine, Tulane University Health Sciences Center, New Orleans, LA, USA
\end{abstract}

Cleft palate is a common birth defect, but its etiopathogenesis is mostly unknown. Several studies have shown that cleft palate has a strong genetic component. Robin sequence consists of three of the following four findings: micrognathia, glossoptosis, obstructive apnea, and cleft palate. While cleft palate is mainly nonsyndromic, about 80 percent of Robin sequence cases are associated with syndromes. Mutations in genes coding for cartilage collagens II and XI, COL2A1, COL11A1 and COL11A2, have been shown to cause chondrodysplasias that are commonly associated with Robin sequence, micrognathia or cleft palate. We therefore analyzed a cohort of 24 patients with nonsyndromic Robin sequence, 17 with nonsyndromic cleft palate and 21 with nonsyndromic micrognathia for mutations in COL11A2. A total of 23 Robin sequence patients were also analyzed for mutations in COL2A1 and COL11A1. We detected two disease-associated mutations in patients with Robin sequence, an Arg to stop codon mutation in COL11A2 and a splicing mutation in COL11A1. Two putatively disease-associated sequence variations were found in COL11A1 in Robin sequence patients, one in COL11A2 in a patient with micrognathia and one in COL2A1 in two patients with Robin sequence. The results showed that sequence variations in these genes can play a role in the etiology of Robin sequence, cleft palate and micrognathia but are not common causes of these phenotypes.

European Journal of Human Genetics (2003) 11, 265-270. doi:10.1038/sj.ejhg.5200950

Keywords: cleft palate; collagen; COL11A1; COL11A2; COL2A1; Robin sequence

*Correspondence: Center for Gene Therapy, Tulane University Health Sciences Center, 1430 Tulane Avenue, SL-99, New Orleans, LA 70112, USA. Tel: +1 5049887709; Fax: +1 5049887710; E-mail: lalako@tulane.edu Received 20 September 2002; revised 4 December 2002; accepted 5 December 2002
Introduction

Oral clefts are among the most common birth defects, affecting approximately 1/500-1/2500 births depending on geographic origin, racial and ethnic backgrounds, and socioeconomic status. ${ }^{1}$ While over 
300 distinct syndromes associated with oral clefts have been identified, most clefts are considered nonsyndromic. $^{2}$

Robin sequence consists of three of the following four findings: micrognathia, glossoptosis, obstructive apnea and cleft palate. ${ }^{3}$ The sequence is associated with numerous syndromes, including Stickler and velocardiofacial syndromes, while nonsyndromic forms comprise about $20 \%$ of all cases. ${ }^{4}$ It has been suggested that a small mandible is the primary defect in nonsyndromic Robin sequence, causing the tongue to be placed posteriorly, which induces cleft palate and upper airway obstruction. However, it has been proposed recently that the cleft palate itself is the primary defect rather than micrognathia, implying that cleft palate and Robin sequence may share the same etiopathogenesis. $^{5}$

Nonsyndromic oral clefting has a complex etiology involving both genetic and environmental factors. ${ }^{1,2}$ Linkage and allelic association studies have yielded contradictory results, although they do indicate some role in clefting for endothelin 1, transforming growth factor $\alpha$ (TGFA), retinoic acid receptor $\alpha(R A R A)$, MSX1 (HOX7), $B C L 3$ and chromosome $6 \mathrm{p}$. Evidence accumulated so far suggests that multiple genes, some of them acting with small effects, are involved in craniofacial development. ${ }^{1,2,6-8}$

Collagen XI is a heterotrimer of $\alpha 1(\mathrm{XI}), \alpha 2(\mathrm{XI})$ and $\alpha 3(\mathrm{XI})$ chains encoded by three distinct genes; COL11A1, COL11A2 and COL2A1. ${ }^{9-11}$ The COL2A1 gene also codes for the $\alpha 1$ chain of collagen II, but the $\alpha 3(\mathrm{XI})$ chain undergoes more extensive post-translational modification than the $\alpha 1$ (II) chain. ${ }^{12}$ Collagens II and XI are present throughout Meckel's cartilage, which provides mechanical support for the developing mandible. ${ }^{13}$ Mutations in the genes coding for collagens XI (COL11A1 and COL11A2) and II (COL2A1) have been shown to cause chondrodysplasias that are commonly associated with micrognathia and palatal defects. ${ }^{9,14-18}$ Among the chondrodysplasias, Stickler syndrome, due to heterozygosity for mutations in COL2A1, is frequently associated with Robin sequence, but most patients with mutations in COL2A1 and COL11A1 also have associated eye phenotypes. Mutations in COL11A2 led to similar chondrodysplasia phenotypes, but patients lack the eye findings. This is because $\alpha 1$ (II) and $\alpha 1$ (XI) are found in the vitreous body of the eye but $\alpha 2$ (XI) is replaced by the $\alpha 2$ chain of collagen $\mathrm{V}$ in this tissue. ${ }^{18}$ For this reason, COL11A2 could be considered the best candidate for nonsyndromic cleft palate.

We screened 24 patients with nonsyndromic Robin sequence, 17 with nonsyndromic cleft palate and 21 with nonsyndromic micrognathia for mutations in COL11A2. COL11A1 and COL2A1 were also analyzed in Robin sequence patients.

\section{Materials and methods Patients}

Cleft patients in Finland have been treated centrally at the Red Cross Hospital for Plastic Surgery since 1948. The Cleft Center has been administered since 1985 by Helsinki University Central Hospital, where 103 patients with Robin sequence were treated during the years 1967-1996. The initial diagnosis was made at the referring hospital in each case. All these patients had cleft palate and a hypoplastic mandible, but breathing difficulties due to glossoptosis was not a consistent finding. We contacted 93 patients by letter. Of them, 33 were willing to participate, but 10 were excluded on account of a confirmed or suspected syndrome. Severe breathing difficulties immediately after birth had been confirmed in seven of the 23 unrelated Robin patients (18-40). None of the patients had eye findings, hearing deficit or skeletal abnormalities consistent with Stickler or Marshall syndrome. Seven of the patients reported that they had a relative who also had a cleft, but in only one case was the affected individual a first-degree relative.

One additional patient with nonsyndromic Robin sequence (62) was referred to us from the USA. The patient underwent a thorough clinical evaluation. An ophthalmological examination at the age of 5 years revealed no vitreous degeneration or detachment and indicated uncorrected 20/20 vision in the right eye and 20/25+ in the left. Hearing was within the normal limits in an audiological examination. There was no evidence of arthropathy, myalgia or loose-jointedness, skeletal radiographs were normal (not shown), and an echocardiogram was normal, with no evidence of mitral valve prolapse (not shown). At 5 years and 6 months of age the patient was in the 50th percentile for weight and height. The 38-year-old father was $175 \mathrm{~cm}$ tall, had a high-arched palate and a small, upturned nose, but no skeletal abnormalities or evidence of osteoarthritis (OA). An audiogram showed a hearing defect at high frequencies in the right ear, most likely attributable to a known history of acoustic trauma. The mother was 38 years old and had myopia, but no skeletal abnormalities or facial dysmorphia.

In all, 17 unrelated patients (1-17) with cleft palate were chosen from among 24 large multiplex families with nonsyndromic cleft palate on whom data had been collected for the Cleft Center of the Helsinki University Hospital over the years 1967-1996. A total of 250 patients had been contacted by letter, and the 24 largest pedigrees had been chosen for further examination. All the patients with nonsyndromic cleft palate had at least one affected relative who had nonsyndromic cleft palate. Patients with a confirmed or strongly suspected syndromic cleft palate were excluded.

In all, 17 patients with nonsyndromic micrognathia were recruited from patient populations at the Center for Craniofacial Disorders and Department of Dentistry at 
Montefiore Medical Center, Bronx, New York, and four from the maxillofacial surgery service at the University Hospitals of Cleveland (patients 41-61). The participants answered a health and family history questionnaire, and any who reported hearing loss, visual problems, joint problems or any other defects suggestive of a syndrome were excluded.

One lateral cephalometric radiograph was taken from each patient recruited in NY, and measurements of mandibular body (gonion-pogonion) and ramus length (articulare-gonion) on these cephalographs were compared with the Bolton standards ${ }^{19}$ for evidence of micrognathia (defined as one or both of these measurements two standard deviations below Bolton's normal values).

Blood samples were obtained from the patients and 150 unrelated Finnish individuals, who had no personal or family history of oral clefting. In addition, one Finnish patient with Marshall syndrome (MP) was included. Parental blood samples were available for patients 20, 22, 26, 33, 35, 39, 62, and MP.

Informed consent was obtained from all the participating subjects.

\section{CSGE analysis and sequencing}

Genomic DNA was used for mutation screening by conformation-sensitive gel electrophoresis (CSGE). ${ }^{20}$ Even though CSGE may not detect all mutations, it has been estimated to have a sensitivity of over $90 \% .^{20}$ The sequences corresponding to the 66 exons of COL11A2, ${ }^{10}$ 66 of the 68 exons (exons 1,3 , and 5 to 68) of COL $11 A 1^{9}$ and the 52 exons of $C O L 2 A 1^{11}$ and exon-flanking sequences were amplified by PCR to obtain products of 182-441 bp. The amplifications and CSGE analysis were performed as described earlier. ${ }^{9,16,20}$ PCR products that showed new or obscure patterns on the CSGE gel were sequenced using an ABI Prism 377 automatic sequenator and the BigDye Terminator Cycle Sequencing Kit (Applied Biosystems).

\section{Analysis of RNA}

Total RNA was isolated from Epstein-Barr virus-transformed lymphoblasts (patients 35 and MP). First-strand cDNA was synthesized with a Superscript Preamplification System (Gibco BRL) using poly-dT primer. PCR amplification of the cDNAs was performed using primers corresponding to exons flanking the sequence of interest, and nested primers were used for the second PCR amplification (Table 1). The PCR amplifications were performed in a volume of $100 \mu \mathrm{l}$, using $2 \mu \mathrm{l}$ of the cDNA/PCR product as a template, $34 \mathrm{pmol}$ of each primer and $5 \mathrm{U}$ of Ampli Taq Gold DNA polymerase. The PCR conditions included one cycle at $95^{\circ} \mathrm{C}$ for $10 \mathrm{~min}$ and 33 cycles at $95^{\circ} \mathrm{C}$ for $40 \mathrm{~s}, 60^{\circ} \mathrm{C}$ for $40 \mathrm{~s}$ and $72^{\circ} \mathrm{C}$ for $45 \mathrm{~s}$, followed by one cycle at $72^{\circ} \mathrm{C}$ for $10 \mathrm{~min}$. The products were analyzed on agarose gels and by sequencing.
Table 1 Primers used for RT-PCR

\begin{tabular}{llll}
\hline Gene & Exon & Primer & Sequence \\
\hline COL2A1 & 14 & 2A1-14F & TGCCTGGTGAAAGAGGACGGAC \\
& 15 & $2 \mathrm{~A} 1-15 \mathrm{~F}$ & GGTGCCCGAGGCAACGATGGT \\
& 16 & $2 \mathrm{~A} 1-16 \mathrm{~F}$ & GTCCTGTCGGTCCTGCTGGT \\
18 & $2 \mathrm{~A} 1-18 \mathrm{R}$ & CAGATCCTTTGGCTCCAGGAATTCC \\
& 19 & $2 \mathrm{~A} 1-19 \mathrm{R}$ & CAGAGGACCAGTTGCCACCTTGAG \\
20 & $2 \mathrm{~A} 1-20 \mathrm{R}$ & TTGCCGCCAGCAATACCAGGTTC \\
COL11A1 & 42 & $11 \mathrm{~A} 1-42 \mathrm{~F}$ & GGCTCACCAGGAGAACGTGGG \\
43 & $11 \mathrm{~A} 1-43 \mathrm{~F}$ & GTCTCCCAGGGCCAGCTGGTCC \\
44 & $11 \mathrm{~A} 1-44 \mathrm{~F}$ GGCAGCAAGGGTGACAAGGG \\
46 & $11 \mathrm{~A} 1-46 \mathrm{R}$ GCTGTCCTCTAGGACCTGGTTC \\
47 & $11 \mathrm{~A} 1-47 \mathrm{R}$ ACCAGGTGGGCCTGGCAGACC \\
48 & $11 \mathrm{~A} 1-48 \mathrm{R}$ GGGACCTTGAGGGCCTCTTGG
\end{tabular}

\section{Results}

CSGE analysis of COL2A1, COL11A1, and COL11A2

All 62 patient samples were analyzed for mutations in the COL11A2 gene, and the 23 patients with Robin sequence for mutations in the COL11A1 and COL2A1 genes as well. Samples that had heteroduplexes on CSGE analysis were sequenced. The observed sequence variations were also searched for in 47 control samples by CSGE analysis, and the ones that were not found in the control set or appeared to be rare were looked for in all 150 control samples, again by CSGE. Six nucleotide substitutions were unique in that they were found only in patients and not in any of the 150 controls (Table 2).

\section{COL11A2 sequence variations}

A single-nucleotide mutation, $\mathrm{C}$ to $\mathrm{T}$, was found in exon 4 of COL11A2 in patient 62, with Robin sequence (Table 2). This changed a codon CGA for Arg to a codon TGA for translation termination, and affected amino acid -310 from the first glycine of the major triple helix. Since the termination codon was in exon 4 of the 66 exon gene, it is likely that the mutant allele cannot produce any functional protein. DNA analysis of the parents indicated that the patient had inherited the mutation from her father. Even though the father did not have Robin sequence, he had a partial phenotype involving a higharched palate and a small, upturned nose which is typically associated with COL11A2 mutations. ${ }^{14-18}$

A $\mathrm{C}$ to $\mathrm{T}$ change converting a codon CGG for Arg to a codon TGG for Trp was found in exon 13 in patient 53, with micrognathia. This affected amino acid -32 in the short N-terminal nontriple helical region between the minor and major triple helical domains. This substitution was interesting because Trp is not found in this region in any of the fibrillar collagens. In addition, Trp substitutions in the $\alpha 2$ and $\alpha 3$ chains of collagen IX have recently been found to be associated with lumbar disc disease. ${ }^{21}$ Patient 53 is now in her 50s, $165 \mathrm{~cm}$ tall, with mild disc degeneration at L4 and early OA in one knee. She had 
Table 2 Unique sequence variations

\begin{tabular}{lcclc}
\hline Gene & Nucleotide change & Predicted consequence & Diagnosis & Patient \\
\hline COL11A1 & IVS31+92T>A & NK & Robin sequence & 20,33 \\
& IVS45+3G $>$ A & NK & Robin sequence, Marshall syndrome & 22, MP \\
COL11A2 & IVS50+3insT & Splicing defect & Robin sequence, Marshall syndrome & 26, Patient 6 \\
& E4+86C $>$ T & R-310X & Robin sequence & 62 \\
COL2A1 & E13+4C $>$ T & R-32W & Micrognathia & 53 \\
& E17+99C $>$ T & NK & Robin sequence & 35,39 \\
\hline
\end{tabular}

${ }^{\mathrm{a}} \mathrm{NK}=$ not known.

${ }^{\mathrm{b}}$ Annunen et $a l_{;}{ }^{9}$ patient 6 .

jaw extension surgery for underbite, and presumably because of this underbite, she had developed temporomandibular joint disease. She currently wears reading glasses but has previously had normal vision. Her palate, uvula, and hearing are normal. Her brother and son have normal jaws. Her niece has a small jaw, but refused DNA analysis.

The analysis also identified sequence changes that implied four other amino-acid substitutions, E-211K, L398P, P830T, and P1236L (data not shown). These are likely to be neutral, because their frequency was similar in the patients and controls.

\section{COL11A1 sequence variations}

Analysis of exon 50 revealed an insertion of a $\mathrm{T}$ at the donor splice site of intron 50, in nucleotide position +3 in patient 26 with Robin sequence. This variation was not present in any of the 150 control samples, but it has been reported earlier in a patient with sporadic Marshall syndrome $^{9}$ (Table 3 ). The present patient was of normal stature $(186 \mathrm{~cm})$ and had mild myopia $(-1.5 /-1.5 \mathrm{D})$ but no hearing defect. All the other family members were unaffected. Neither of the parents had the mutation, and therefore, this patient also had a sporadic mutation. Even though RNA was not available to enable the consequence in terms of splicing to be examined, the mutation is predicted to result in a splicing defect. ${ }^{9,22}$

The second unique sequence variation, IVS45+3G $>A$, was in COL11A1 in patient 22, with Robin sequence (Table 2 ). The mother was found to have the same sequence change. She had died of diabetic complications at the age of 28. Neither parent of patient 22 had cleft palate or any other craniofacial malformations, but the son of the proband's paternal aunt had cleft lip and palate. We had previously identified the same sequence variation in a patient with Marshall syndrome (MP) who had Robin sequence, severe myopia (-20/-20D), midfacial hypoplasia, hyperflexible finger joints, and a mild conductive hearing defect. The father of this latter patient was found to have the same mutation. He had no cleft palate but had a small mandible at birth. He also had mild myopia (-2.75/ $-2.5 \mathrm{D})$. The paternal grandmother had myopia $(-6 /-6 \mathrm{D})$
Table 3 Comparison of the phenotypes of Marshall syndrome and Robin sequence patients with IVS50+3insT in COL11A1

\begin{tabular}{lcc}
\hline Clinical findings & \multicolumn{2}{c}{ Patient } \\
& $\begin{array}{c}\text { Robin sequence } \\
(26)\end{array}$ & Marshall $^{\text {a }}$ \\
\hline Cleft palate & + & + \\
Micrognathia & + & - \\
$\begin{array}{l}\text { Breathing difficulties } \\
\text { at birth (D) }\end{array}$ & + & $?$ \\
Myopia (D) & $-1.5 /-1.5$ & $-18 /-20$ \\
$\begin{array}{l}\text { Hearing defect } \\
\text { Midfacial hypoplasia }\end{array}$ & No & Mixed type \\
Stature & No & + \\
& Normal & short \\
\hline
\end{tabular}

${ }^{\mathrm{a} A n n u n e n}$ et al; $^{9}$ patient 6.

and hyperflexible joints, but she did not have a cleft palate or hearing impairment. Both $G$ and $A$ nucleotides are commonly observed in position 3 of a donor splice site, but there are several examples of A to G mutations in this position causing aberrant splicing. ${ }^{23}$ RT-PCR analysis failed to indicate a splicing defect (data not shown). To exclude the possibility of nonsense-mediated mRNA decay by means of a cryptic splice site, exon $5+9 \mathrm{~T}>\mathrm{C}$ polymorphism and exon $63+162 \mathrm{C}>\mathrm{T}$ polymorphisms were analyzed. Sequencing showed that both polymorphisms were present in the mRNA, and thus both alleles were expressed. The analysis could not rule out the possibility of a quantitative splicing defect, however.

One nucleotide substitution, IVS31+92T $>A$, was found in two patients with Robin sequence (20 and 33) but not in any of the 150 controls tested. Analysis of the parents' DNA revealed that both patients had inherited the substitution from one of their parents. Since the parents and other family members did not have any cleft defects, the substitution is not likely to be pathological, but we cannot rule out the possibility that it may predispose carriers to the phenotype.

The analysis also identified three amino-acid substitutions, L795P, P1007S, and P1008A, but these were not associated with clefting, as they were found equally frequently in the patient and control samples. 


\section{COL2A1 sequence variations}

A unique nucleotide change was found in exon 17 of COL2A1 in two unrelated patients with Robin sequence (35 and 39), the last nucleotide $C$ of this exon being replaced with T. This did not change the amino acid encoded. The father of patient 35 was heterozygous for the same change, but no one else in this family had cleft palate except the patient. The mother of patient 39 was found to be homozygous for the nucleotide change and had nonsyndromic cleft palate. She had three unaffected sisters and a brother. All four of her grandparents originated from the same region. Since RT-PCR analysis did not reveal any splicing defects and showed that both alleles were expressed, this mutation is not likely to be pathological, but it may be a predisposing, as three of the four individuals with this nucleotide variation had a clefting defect.

The analysis identified only one amino-acid substitution, V1131I, which is likely to be neutral, as it was found to be present equally frequently in the patients and controls.

\section{Discussion}

Mutations in the collagens II and XI genes cause syndromes that are often associated with cleft palate, Robin sequence and micrognathia, but it is possible that they may also cause nonsyndromic conditions. We identified here two disease-associated mutations in patients with Robin sequence, an $\mathrm{R}$ to $\mathrm{X}$ mutation in COL11A2 and a splicing mutation in COL11A1. Additionally, two putatively disease-predisposing mutations were found in COL11A1 in Robin sequence patients, one in COL11A2 in a patient with micrognathia and one in COL2A1 in two patients with Robin sequence.

It has been proposed that a major clefting locus may be contained in chromosome $6 \mathrm{p}^{2}$ and COL11A2 is located close, in head-to-tail orientation, to a gene for the retinoic acid receptor $\beta, R X R-\beta$, on $6 \mathrm{p} 21.2 .^{10}$ The corresponding mouse genes have the same orientation and it has been demonstrated that some transcripts from $R x r-\beta$ extend into Col11a2. ${ }^{24}$ Excess administration of retinoic acids to pregnant women is known to cause micrognathia and cleft palate, ${ }^{25,26}$ possibly by upregulating the expression of $R X R-\beta$, resulting in downregulation of COL11A2 and thereby producing a phenotype similar to those seen in connection with mutations in collagen XI genes. ${ }^{14-18}$ This hypothesis is supported by a recent finding that loss of function mutations in COL11A2 cause otospondylomegaepiphyseal dysplasia (OSMED), a recessively inherited phenotype typically associated with Robin sequence. ${ }^{16}$ Furthermore, one of the well-defined chromosomal regions for cleft susceptibility in the mouse is in or near the $\mathrm{H} 2$ locus, which is homologous to the human HLA locus on $6 \mathrm{p}$ and includes susceptibility for both corticosteroid-induced and vitamin A-enhanced cleft palate. ${ }^{2}$
We identified here a stop codon mutation in COL11A2 in a patient with nonsyndromic Robin sequence. The father of the patient, who had the same mutation, had a high-arched palate and a small, upturned nose, but no cleft palate. Thus, the mutation caused nonsyndromic Robin sequence in the daughter, but was not fully penetrant in the father. Even though Robin sequence is associated with OSMED, which is typically caused by loss of function mutations in COL11A2, it is likely that COL11A2 haploinsufficiency can predispose to Robin sequence. This is supported by the previous findings that some carriers in OSMED families had a partial phenotype. ${ }^{16}$ Furthermore, the role of COL11A2 in nonsyndromic conditions is supported by a recent finding that mutations in this gene can cause nonsyndromic hearing loss. ${ }^{27}$

Another mutation, IVS50+3insT in COL11A1, was found in two patients, one with Robin sequence and the other with Marshall syndrome. ${ }^{9}$ The finding that a mutation can result in two different but overlapping phenotypes is not surprising, and there are several examples of identical mutations in COL1A1 causing osteogenesis imperfecta that can vary from mild to lethal. ${ }^{28}$

It is likely that a single genetic factor is not in itself enough to result in clefting, and that other, as yet unknown genetic or environmental factors modify the effects of mutations that predispose to clefting. It is thus possible that the unique sequence variations found here may be associated with the phenotypes but cannot alone induce clefting. This hypothesis is supported by the findings that nonsyndromic oral clefting is multifactorial and involves both genetic and environmental factors ${ }^{1,2}$ and that the concordance for nonsyndromic cleft palate in monozygotic twins is only $23.5 \%{ }^{29}$

Even though disease-associated alterations in the three candidate genes were not found in the majority of patients, we can conclude that sequence variations in these genes may cause or predispose to nonsyndromic Robin sequence, cleft palate, and micrognathia in some cases.

\section{Acknowledgements \\ We thank all the patients and their families who participated in this study, and also Mrs Aira Harju and Ms Minta Lumme for their expert technical assistance. This work was partially supported by the Academy of Finland (grants to LA-K and JK), the Arthritis Foundation, the European Commission Grant (QLRT2001-02188), the Louisiana Gene Therapy Research Consortium (New Orleans, LA) and HCA-The Healthcare Company (Memphis, TN) (to LA-K), the Helsinki University Hospital Research Funds, the Sigrid Juselius Foundation (to JK) and NIH grant HD22657 (to DC and DK).}

\section{References}

1 Schutte BC, Murray JC: The many faces and factors of orofacial clefts. Hum Mol Genet 1999; 8: 1853-1859.

2 Murray JC: Face facts: genes, environment and clefts. Am J Hum Genet 1995; 57: 227-232. 
3 Robin P: La chute de la base de la langue considerée comme une nouvelle cause de gêne dans la respiration naso-pharyngienne. Bull Acad Natl Med (Paris) 1923; 89: 37-41.

4 Shprintzen RJ: The implications of the diagnosis of Robin sequence. Cleft Palate Craniofac J 1992; 29: 205-209.

5 Marques IL, Barbieri MA, Bettiol H: Etiopathogenesis of isolated Robin sequence. Cleft Palate Craniofac J 1998; 35: 517-525.

6 Prescott NJ, Lees MM, Winter RM, Malcolm S: Identification of susceptibility for nonsyndromic cleft lip with or without cleft palate in a two stage genome scan of affected sibpairs. Hum Genet 2000; 106: 345-350.

7 Sakata Y, Tokunaga K, Yonehara Y et al: Significant association of HLA-B and HLA-DRB1 alleles with cleft lip with or without cleft palate. Tissue Antigens 1999; 53: 147-152.

8 Wong FK, Hagberg C, Karsten A et al: Linkage analysis of candidate regions in Swedish nonsyndromic cleft lip with or without cleft palate families. Cleft Palate Craniofac J 2000; 37: 357-362.

9 Annunen S, Körkkö J, Czarny M et al: Splicing mutations of 54 bp exons in the COL11A1 gene cause Marshall syndrome but other mutations cause overlapping Marshall/Stickler phenotypes. Am J Hum Genet 1999; 65: 974-983.

10 Vuoristo MM, Pihlajamaa T, Vanderberg P, Prockop DJ, Ala-Kokko $\mathrm{L}$ : The human COL11A2 gene structure indicates that the gene has not evolved with the genes for the major fibrillar collagens. J Biol Chem 1995; 270: 22873-22881.

11 Ala-Kokko L, Kvist A-P, Metsäranta $\mathrm{M}$ et al: Conservation of the sizes of 53 introns and over 100 intronic sequences for the binding of common transcription factors in the human and mouse genes for type II procollagen (COL2A1). Biochem J 1995; 308: 923-929.

12 Eyre D, Wu JJ: Type XI or $1 \alpha 2 \alpha 3 \alpha$ collagen; in Mayne R, Burgeson $\mathrm{RE}$, (eds): Structure and function of collagen types. Academic Press, New York: 1987, pp 261-281.

13 Chung KS, Park HH, Ting K et al: Modulated expression of type X collagen in the Meckel's cartilage with different developmental fates. Dev Biol 1995; 170: 387-396.

14 Spranger J: The type XI collagenopathies. Pediatr Radiol 1998; 28: 745-750.

15 Sirko-Osadsa DA, Murray MA, Scott JA, Lavery MA, Warman ML, Robin NH: Stickler syndrome without eye involvement is caused by mutations in COL11A2 the gene encoding the $\alpha 2$ (XI) chain of type XI collagen. J Pediatr 1998; 132: 368-371.
16 Melkoniemi M, Brunner HG, Manouvrier S et al: Autosomal recessive disorder otospondylomegaepiphyseal dysplasia is associated with loss-of-function mutations in the COL11A2 gene. Am J Hum Genet 2000; 66: 368-377.

17 Pihlajamaa T, Prockop DJ, Faber J et al: Heterozygous glycine substitution in the COL11A2 gene in the original patient with the Weissenbacher-Zweymüller syndrome with heterozygous OSMED (nonocular Stickler syndrome). Am J Med Genet 1998; 80: $115-120$.

18 Myllyharju J, Kivirikko KI: Collagens and collagen-related diseases. Ann Med 2001; 33: 7-21.

19 Broadbent $\mathrm{BH}$, Broadbent Jr BH, Golden WH: Bolton standards of dentofacial developmental growth. Saint Louis: CV Mosby Co., 1975.

20 Körkkö J, Annunen S, Pihlajamaa T, Prockop DJ, Ala-Kokko L: Conformation sensitive gel electrophoresis for simple and accurate detection of mutations: comparison with denaturing gradient gel electrophoresis and nucleotide sequencing. Proc Natl Acad Sci USA 1998; 95: 1681-1685.

21 Ala-Kokko L: Genetic risk factors for lumbar disc disease. Ann Med 2002; 34: 42-47.

22 Cartegni J, Chew SL, Krainer AR: Listening to silence and understanding nonsense: exonic mutations that affect splicing. Nat Rev Genet 2002; 3: 285-298.

23 Zeniou M, Pannetier S, Fryns JP, Hanauer A: Unusual splice-site mutations in the RSK2 gene and suggestion of genetic heterogeneity in Coffin-Lowry syndrome. Am J Hum Genet 2002; 70: 1421-1433.

24 Vanderberg P, Vuoristo MM, Ala-Kokko L, Prockop DJ: The mouse col11a2 gene. Some transcripts from the adjacent $\operatorname{rxr}-\beta$ gene extend into the col11a2 gene. Matrix Biol 1996; 15: 359-367.

25 Benke PJ: The isotretionin teratogen syndrome. JAMA 1984; 251: 3267-3269.

26 Lammer EJ, Chen DT, Hoar RM et al: Retinoic acid embryopathy. N Eng J Med 1995; 313: 837-841.

27 McGuirt WT, Prasad SD, Griffith AJ et al: Mutations in COL11A2 cause nonsyndromic hearing loss (DFNA13). Nat Genet 1999; 23: 413-419.

28 Körkkö J, Kuivaniemi $\mathrm{H}$, Paassilta $\mathrm{P}$ et al: Two new recurrent nucleotide mutations in the COL1A1 gene in four patients with osteogenesis imperfecta: about one-fifth are recurrent. Hum Mut 1997; 9: 148-156.

29 Fraser FC: The genetics of cleft lip and cleft palate. Am J Hum Genet 1970; 22: 336-352. 